\title{
Three-Dimensional Direct Numerical Simulation of Turbulent Lean Premixed Methane Combustion with Detailed Kinetics
}

\author{
A. J. Aspden ${ }^{\mathrm{a}, \mathrm{b}}$, M. S. Day ${ }^{\mathrm{b}}$, J. B. Bell ${ }^{\mathrm{b}}$ \\ ${ }^{a}$ Mathematical Sciences, University of Southampton, Southampton, Hampshire, SO17 1BJ, \\ UK \\ ${ }^{b}$ Center for Computational Sciences and Engineering, Lawrence Berkeley National \\ Laboratory, 1 Cyclotron Road, MS50A-1148, Berkeley, CA 94720, USA
}

\begin{abstract}
The interaction of maintained homogeneous isotropic turbulence with lean premixed methane flames is investigated using direct numerical simulation with detailed chemistry. The conditions are chosen to be close to those found in atmospheric laboratory experiments. As the Karlovitz number is increased from 1 to 36 , the preheat zone becomes thickened, while the reaction zone remains largely unaffected. A negative correlation of fuel consumption with mean flame surface curvature is observed. With increasing turbulence intensity, the chemical composition in the preheat zone tends towards that of an idealised unity Lewis number flame, which we argue is the onset of the transition to distributed burning, and the response of the various chemical species is shown to fall into broad classes. Smaller-scale simulations are used to isolate the specific role of species diffusion at high turbulent intensities. Diffusion of atomic hydrogen is shown to be related to the observed curvature correlations, but does not have significant consequential impact on the thickening of the preheat zone. It is also shown that susceptibility of the preheat zone to thickening by turbulence is related to the 'global' Lewis number (the Lewis number of the deficient reactant); higher global Lewis number flames tend to be more prone to thickening.
\end{abstract}

Keywords: Turbulent premixed flames, direct numerical simulation, detailed chemistry, low Mach number flow, adaptive mesh refinement

\section{Introduction}

Direct numerical simulation with detailed kinetics provides a powerful tool for studying the fundamentals of turbulent combustion, and for the development

\footnotetext{
${ }^{*}$ Corresponding author: A. J. Aspden (a.j.aspden@soton.ac.uk)

Email addresses: a.j.aspden@soton.ac.uk (A. J. Aspden), msday@lbl.gov (M. S. Day), jbbell@lbl.gov (J. B. Bell)
}

Preprint submitted to Combustion and Flame

January 22, 2016

(C) 2016. This manuscript version is made available under the Elsevier user license http://www.elsevier.com/open-access/userlicense/1.0/ 
and validation of turbulent flame models for engineering applications. As part 5 of the effort to develop clean-burning efficient systems to meet growing global energy demands, there is significant interest in lean premixed methane combustion. Burning under lean conditions reduces the exhaust gas temperatures, and consequently, thermal $\mathrm{NO}_{\mathrm{x}}$ emissions. In this paper, three-dimensional direct numerical simulations of lean premixed methane combustion with detailed ki-

10 netics are presented over a range of turbulent intensities representative of the conditions found in typical experimental studies. The focus of the study is on the interactions between turbulence and combustion, and how the flame is affected as turbulent intensity increases. The goal is to provide a set of benchmark DNS data that can be used both to study basic flame physics and to provide a baseline for evaluation of turbulent combustion models.

There is a growing body of work in the literature that deals with turbulent premixed methane flames with detailed kinetics. The earliest work in this area investigated two-dimensional configurations using a detailed $\mathrm{C} 1$ mechanism 1, 2, 3, 4. Bell et al. [5 produced the first simulation of a turbulent methane

20 flame in three dimensions with moderate-fidelity kinetics using DRM-19, which is a simplified mechanism derived from GRIMech 1.2. Simulations using DRM19 for a variety of experimental configuration have been presented in [6, 7]. Sankaran et al. [8] performed a DNS of the lean premixed preheated methane flame stabilised on a slot burner using a skeletal mechanism with 13 species 25 derived from GRIMech 1.2. More recently, Aspden et al. 9] and Carlsson et al. 10, 11 presented DNS of lean premixed methane flames at high turbulence levels near to the transition to distributed burning. The simulations presented in the present paper were conducted using GRIMech 3.0 without the emissions chemistry [12, 13].

Here lean premixed methane flames are considered at an equivalence ratio of $\varphi=0.7$, for which the Lewis number of the fuel is close to unity; the Lewis number of the fuel (i.e. the deficient reactant) will be referred to as the global Lewis number. The characterisation of the turbulence in terms of the unstrained laminar flame properties is given by the Karlovitz and Damköhler numbers, which are defined by

$$
\mathrm{Ka}^{2}=\frac{\Upsilon^{3}}{\Lambda}=\frac{u^{3}}{s_{F}^{3}} \frac{l_{F}}{l} \quad \text { and } \quad \mathrm{Da}=\frac{\Lambda}{\Upsilon}=\frac{s_{F}}{u} \frac{l}{l_{F}}
$$

30 where $u$ and $l$ are the turbulent rms velocity fluctuation and integral length scale, respectively, and $s_{F}$ and $l_{F}$ are the unstrained flat laminar flame speed and width, respectively, $\Lambda=l / l_{F}$ and $\Upsilon=u / s_{F}$. The Damköhler number represents the ratio of chemical and integral length time scales. The Karlovitz number represents the ratio of Kolmogorov and chemical time scales, and can be thought

35 of as a measure of the strength of the turbulence relative to the flame. From the point of view of turbulence-flame interactions, the more relevant parameter is the Karlovitz number; fixing Ka effectively fixes the energy dissipation rate $\varepsilon=u^{3} / l$ and the Kolmogorov length scale $\eta=\left(\nu^{3} / \varepsilon\right)^{1 / 4}$, and therefore the turbulence that interacts with the flame. In the present paper, Karlovitz numbers between 
$40 \quad 1$ and 36 are presented. Turbulence is generated by incorporating a forcing term into the momentum equations that can be adjusted to match desired turbulence properties using our well-established approach [14, 15, 16]. The same domain size is used for all simulations, which results in a fixed integral length scale, approximately one-tenth the width of the domain, and four times the thermal

45 thickness of the unstrained laminar flame (i.e. $\Lambda=4$ ). The conditions that are considered are typical of those encountered in a range of atmospheric laboratory scale experiments; i.e. turbulent velocity fluctuations on the order of a few metres per second and an integral length scale on the order of a few millimetres.

The computational methodology and simulation configuration are presented

${ }_{50}$ in section 2. An overview of the simulations and flame response to increasing Karlovitz number are then presented in section 3.1, followed by joint probability density functions of flame speed and curvature in section 3.2. Two-dimensional slices through the three-dimensional data present changes in chemical composition in section 3.3 , and thickening of the preheat zone is examined in sec55 tion 3.4. The chemical composition is explored further through conditional means of species molar concentration in section 3.5. The effect of diffusive processes are investigated by running further small-scale simulations in section 4 . where species diffusion coefficients are artificially adjusted. In particular, several key observations are shown to be related to the high mobility of atomic hydro-

60 gen. This is demonstrated using slices (section 4.1), joint probability density functions (section 4.2), flame thickening (section 4.3), and conditional means of molar concentrations (section 4.4). The paper closes with a summary of the conclusions (section 5).

\section{Computational Methodology}

The simulations presented here are based on a low Mach number formulation of the reacting flow equations. The methodology treats the fluid as a mixture of perfect gases. A mixture-averaged model is used for differential species diffusion, ignoring Soret and Dufour effects, as well as neglecting gravity and radiative transport processes. With these assumptions, the low Mach number equations for an open domain are

$$
\begin{aligned}
\frac{\partial(\rho \boldsymbol{u})}{\partial t}+\nabla \cdot(\rho \boldsymbol{u u}) & =-\nabla \pi+\nabla \cdot \tau+\rho \boldsymbol{F}, \\
\frac{\partial\left(\rho Y_{i}\right)}{\partial t}+\nabla \cdot\left(\rho Y_{i} \boldsymbol{u}\right) & =\nabla \cdot\left(\rho \mathcal{D}_{i} \nabla Y_{i}\right)-\rho \dot{\omega}_{i}, \\
\frac{\partial(\rho h)}{\partial t}+\nabla \cdot(\rho h \boldsymbol{u}) & =\nabla \cdot\left(\frac{\lambda}{c_{p}} \nabla h\right)+\sum_{i} \nabla \cdot\left[h_{i}\left(\rho \mathcal{D}_{i}-\frac{\lambda}{c_{p}}\right) \nabla Y_{i}\right],
\end{aligned}
$$

where $\rho$ is the density, $\boldsymbol{u}$ is the velocity, $Y_{i}$ is the mass fraction of species $i$, $h$ is the specific enthalpy of the gas mixture, $T$ is the temperature, and $\dot{\omega}_{i}$ is the net destruction rate for species $i$ due to chemical reactions. Also, $\lambda$ is the thermal conductivity, $\tau$ is the stress tensor, $c_{p}$ is the specific heat of the ${ }_{75}$ mixture, and $h_{i}(T)$ and $\mathcal{D}_{i}$ are the specific enthalpy and mixture-averaged 
diffusion coefficients of species $i$, respectively. Here, $\boldsymbol{F}$ is a long-wavelength forcing term designed to establish and maintain turbulence with the desired properties. These evolution equations are supplemented by an equation of state for a perfect gas mixture.

The basic discretisation combines a symmetric operator-split treatment of chemistry and transport with a density-weighted approximate projection method. The projection method incorporates the equation of state by imposing a constraint on the velocity divergence. The resulting integration proceeds on the time scale of the velocity with advective transport treated explicitly. Faster 85 diffusion and chemistry processes are treated time-implicitly. This integration scheme is embedded in a parallel adaptive mesh refinement algorithm framework based on a hierarchical system of rectangular grid patches. The complete integration algorithm is second-order accurate in space and time, and discretely conserves species mass and enthalpy. The reader is referred to [17 for details. The chemical kinetics and transport are modelled using the GRIMech 3.0 methane mechanism [12, 13] with the nitrogen chemistry removed. The resulting mechanism consists of 35 species with 217 reactions.

The overall numerical scheme is known to converge with second-order accuracy, and the ability of the scheme to perform direct numerical simulation 95 was examined in [14. An effective Kolmogorov length scale was formulated, which measures the Kolmogorov length scale that actually results from a simulation at a given resolution. The main simulations presented here all have an effective Kolmogorov length scale within $0.9 \%$ of the analytical value, and so the turbulence can be considered to be sufficiently-well resolved. The narrowest intermediate chemical species have a thickness (defined using the points at half of the peak value) that is spanned by approximately six computational cells; the profiles can be well reproduced with half the resolution used here, and so the chemistry can also be considered well-resolved.

\subsection{Simulation Configuration}

105 Three-dimensional simulations were conducted for four statistically-stationary downward-propagating flames in a high aspect ratio domain, with periodic lateral boundary conditions, a free-slip base and outflow at the top. The turbulent background velocity field was maintained through the source term in the momentum equations following 14 . This results in a time-dependent zero-mean turbulent velocity field. It was shown in [14] that this approach gives approximately 10 integral length scales across the domain width. The domain width is $L=2.64 \mathrm{~cm}$, which results in an integral scale of $l \approx 2.6 \mathrm{~mm}$. An inert calculation was run to establish the turbulence at reduced expense, and the reacting flow simulation was initialised by superimposing a laminar flame solution onto 115 the turbulent velocity field.

The unburned fuel mixture was taken to have a temperature of $298 \mathrm{~K}$ and standard atmospheric pressure, and the equivalence ratio was taken to be $\varphi=$ 0.7 , which gives a global Lewis number of approximately unity. The unstrained laminar flame speed is approximately $18.9 \mathrm{~cm} / \mathrm{s}$, and the thermal thickness, 
microns. The adiabatic flame temperature is $1842 \mathrm{~K}$. At the specified resolution there are over 25 computational cells across the thermal flame thickness, which is more than sufficient to resolve the flame structure. For these conditions, the integral scale is four times the flame thickness for all of the cases. The rms velocity fluctuations were varied from approximately 1.6 to 17 times the unstrained laminar flame speed. Details of the four cases considered are given in table 1, and the position of these flames on the premixed regime diagram are shown in figure 1. Note that the highest turbulent intensity is less than the value used in [16]; however, the integral length scale is about 8 times larger,

130

The base grid in each case had a cross section of $256 \times 256$, with two levels of refinement used once the flame had become established, giving an effective resolution of $1024 \times 1024$ in cross section. The simulations at $\mathrm{Ka}=1$ and 4 had a domain that was four times longer in the direction of propagation than each of the lateral directions, and the simulations at $\mathrm{Ka}=12$ and 36 were eight times longer. A significant buffer region of refinement was used ahead of the flame to ensure that the turbulence was well-developed (i.e. the cascade had reached the high wavenumber modes) as established in [16]. A particular advantage of adaptive mesh refinement is that the turbulent flame can be established on the base grid, and the full resolution simulations run for a short period time for data collection. The data presented for the four Karlovitz numbers were taken after 4.5, 11, 14 and 24 turbulent eddy turnover times, respectively, measured from the beginning of the simulation.

\section{Analysis of Simulations}

Before examining the turbulent flames in detail, an overall characterisation of the behaviour is given, including turbulent flame speeds and correlations with curvature. Slices of molar concentration show an apparent thickening of the flame, especially in the preheat zone, which is quantified using a thickening factor. Finally, the effect of turbulence on the chemical composition is investigated using conditional means of species molar concentrations.

\subsection{Overview}

In figure 2, representative slices of temperature and fuel consumption rate are presented for the four cases; the entire width of the domain is shown, but only 1.5 domain widths are shown in the direction of flame propagation. Each panel is normalised by the corresponding value from the unstrained laminar flame. As expected, as Ka increases, there is an increase in both flame surface area and flame wrinkling; there are a greater number of features with high curvature. There appears to be only a slight increase in fine scale structure between $\mathrm{Ka}=12$ and 36, suggesting that the large-scale interactions are saturating.

Global consumption-based flame speed (defined as the volume integral of fuel consumption over the domain divided by the cross-sectional area of the domain) normalised by the unstrained laminar flame speed is shown in figure 3 as a 
function of both time and Karlovitz number for the four cases. The simulations do not cover a sufficiently long time to obtain a statistically converged mean flame speed, but nominal averages are denoted by crosses, and the vertical lines show the entire range of values obtained during the averaging period. Even at $\mathrm{Ka}=1$, the turbulent flame speed is over four times that of the unstrained laminar flame, and increases with Karlovitz number (as does the spread), and possibly levels off around $\mathrm{Ka}=36$ at just over ten times faster than the un-

170 strained laminar flame. The high turbulent flame speed results from the increase in flame surface area; the circle and square in figure 3 (b) respectively denote the consumption-based flame speed and normalised flame surface area (based on the flame surface area of the isotherm corresponding to the peak heat release) at the same time point. Note that such an increase in flame surface area is partly due to the large domain size and integral length scale $\left(L=40 l_{F}\right.$ and $\left.l=4 l_{F}\right)$.

\subsection{JPDFs of Flame Speed and Curvature}

At $\varphi=0.7$, the global Lewis number is approximately unity, which is reflected in the fuel consumption plot in figure 2, which is essentially uniform along the flame front for $\mathrm{Ka}=1$ and 4 . At $\mathrm{Ka}=12$ and 36 , the turbulent intensity becomes sufficiently strong to disrupt the preheat zone and there is variability in both the fuel consumption rate and the heat release along the flame front. Of particular note is a reduction of fuel consumption rate and heat release in regions of high positive mean curvature (where the centre of curvature is in the products). To quantify this effect, the flame surface was identified with the $1484 \mathrm{~K}$ isotherm, which is the temperature corresponding to peak fuel consumption in the flat laminar flame. A local coordinate system is then constructed in a neighbourhood of the flame by following integral curves of $\nabla T$, following the procedure detailed in [18. Given a fine triangularisation of the flame surface, a prism (with triangular cross section) can be constructed extending from the triangle that follows the local coordinate lines in both directions. Integration of the fuel consumption over these prisms allows a local consumption-based flame speed to be defined,

$$
s_{T}^{c}=\frac{1}{\left(\rho Y_{\mathrm{CH}_{4}}\right)_{\text {reac }} A_{\text {ref }}} \int_{\Omega} \rho \dot{\omega}_{\mathrm{CH}_{4}} \mathrm{~d} \Omega,
$$

where the suffix reac denotes the reactants, $\Omega$ is prism volume, and $A_{\text {ref }}$ is the area of intersection between the $\Omega$ and the flame surface. In figure 4 .JPDFs of $s_{T}^{c}$ and mean curvature $M$ are plotted for the four cases, where $M \equiv \kappa_{1}+\kappa_{2}$ 180 on the flame surface for $\kappa_{1}$ and $\kappa_{2}$ the two principal curvatures. At $\mathrm{Ka}=$ 1 , the JPDF is essentially flat, indicating that $s_{T}^{c}$ is insensitive to curvature. As Ka increases, the JPDF broadens, reaffirming the increased occurrence of highly curved structures. An increasingly strong negative correlation emerges with increasing Ka, illustrating an increasing dependence of the local flame structure on curvature; in regions of high positive curvature, there is a decrease in the local consumption-based burning speed, and conversely in regions of high negative curvature, there is an increase in $s_{T}^{c}$. The normalised flame speed 
is approximately $5 \%$ higher than that of the unstrained laminar flame, so the five-to-tenfold increase in overall consumption-based turbulent flame speed in

\subsection{Slices of molar concentrations}

Slices of density and molar concentration for eight species for the same slice as figure 2 at $\mathrm{Ka}=36$ are presented in figure 5 , all of the slices have been normalised by corresponding values from the unstrained laminar flame. The

195 different species have been chosen as representative of different classes of species or because they present interesting behaviour, and the same species will be used in considering conditional means below; all of the species have been included as supplementary material. The species classifications used are reactants $\left(\mathrm{CH}_{4}\right.$ and $\left.\mathrm{O}_{2}\right)$, products $\left(\mathrm{CO}_{2}\right.$ and $\left.\mathrm{H}_{2} \mathrm{O}\right)$, stable intermediate species $\left(\mathrm{H}_{2}, \mathrm{C}_{2} \mathrm{H}_{6}, \mathrm{C}_{2} \mathrm{H}_{4}\right.$, $\mathrm{C}_{3} \mathrm{H}_{8}, \mathrm{C}_{2} \mathrm{H}_{2}, \mathrm{CH}_{3} \mathrm{OH}, \mathrm{HCCOH}, \mathrm{CH}_{2} \mathrm{O}, \mathrm{CH}_{3} \mathrm{CHO}, \mathrm{CH}_{2} \mathrm{CO}$ and $\mathrm{CO}$ ), species with low-temperature activity $\left(\mathrm{HO}_{2}, \mathrm{H}_{2} \mathrm{O}_{2}, \mathrm{CH}_{3} \mathrm{O}, \mathrm{C}_{3} \mathrm{H}_{7}\right.$ and $\left.\mathrm{CH}_{2} \mathrm{CHO}\right)$, hightemperature radicals with non-zero concentrations above $1800 \mathrm{~K}(\mathrm{H}, \mathrm{O}$, and $\mathrm{OH})$, and the other high-temperature radicals $\left(\mathrm{HCO}, \mathrm{C}_{2} \mathrm{H}_{5}, \mathrm{CH}_{3}, \mathrm{CH}_{2}-\mathrm{S}, \mathrm{CH}_{2}\right.$, $\mathrm{HCCO}, \mathrm{C}_{2} \mathrm{H}_{3}, \mathrm{C}_{2} \mathrm{H}, \mathrm{CH}_{2} \mathrm{OH}, \mathrm{CH}$ and $\mathrm{C}$ ).

${ }_{205}$ It is immediately apparent from the slices of density and fuel (figure 5(a,b); representative of reactants) that there is significant broadening of the preheat zone; there is light red/orange several integral length scales from the flame surface; this is suggestive that turbulence is able to mix species in the preheat zone, and will be considered in more detail below. A similar picture is presented by ${ }_{210} \mathrm{CO}_{2}$ (figure 5(c); representative of products) where pale blue regions can be seen far from the flame surface. Again, for ethane (figure 5(d); representative of stable intermediates), there appears to be pale blue regions extending into the preheat zone, but the maximum concentration exceeds that of the unstrained laminar flame (highlighted in black); perhaps suggestive of more intense or nar215 rower burning. $\mathrm{HO}_{2}$ and $\mathrm{CH}_{3} \mathrm{O}$ (figure $5(\mathrm{e}, \mathrm{f}$ ); representative of species that have low-temperature activity) present strong curvature dependence (as previously reported [5]), where concentrations drop almost to zero in regions of high positive curvature, and far exceed the peak value of the unstrained laminar flame in regions of negative curvature (again, highlighted in black). Molecular and 220 atomic hydrogen (figure $5(\mathrm{~g}, \mathrm{~h})$ ) are highly diffusive, and are considered separately from the other species of the same type. The former is both highly mobile and relatively stable at low temperatures, which is reflected by the diffuse image. Atomic hydrogen is a high-temperature radical, and shows broad regions that exceed the peak value in the unstrained laminar flame (once again, in black).

225 Finally, HCO (figure 5 (i); representative of the other high-temperature radicals) naturally presents a narrow profile, but also a slight reduction in regions of high positive curvature.

\subsection{Thickening of the preheat zone}

To quantify the apparent broadening of the preheat zone, a thickening factor is defined by taking the gradient of the temperature field, evaluating the conditional mean as a function of temperature (the conditioning variable is denoted 
by $\xi$ ), and normalising by the corresponding profile from the $\mathrm{Ka}=1$ flame,

$$
\Theta_{n}(T)=\frac{<\nabla T(\xi) \mid \xi=T>_{\mathrm{Ka}=1}}{<\nabla T(\xi) \mid \xi=T>_{\mathrm{Ka}=n}} .
$$

The Ka $=1$ flame was used to normalise the profile (in preference to the unstrained laminar flame) because it permits comparison with hydrogen flames from a previous study [19. The unstrained laminar profiles for low Lewis number flames are inappropriate because they fail to take into account the effects of the thermodiffusive instability, effects that can accounted for by normalising by the $\mathrm{Ka}=1$ flame. Further details of the issues characterising low Lewis number 235 flames can be found in 20. Note that this will make almost no difference when the global Lewis is close to unity or higher because the $\mathrm{Ka}=1$ will be close the unstrained laminar profile.

The thickening factor $\Theta_{n}$ is presented in figure 6 for $\mathrm{Ka}=4,12$ and 36, where the temperature has been normalised by the adiabatic flame temperature 240 (as a very loose reference, each step of 0.2 corresponds to just over $300 \mathrm{~K}$ ). At $\mathrm{Ka}=4, \Theta_{4} \approx 1$ throughout the flame; there is little, if any, thickening (and by implication one would not expect substantial difference if normalising by the unstrained laminar flame). As Ka increases, there is an increase in the thickening factor at low temperatures; for normalised temperature below

2450.3 (corresponding to approximately $760 \mathrm{~K}$ ) the flame is at least $50 \%$ thicker at the higher Karlovitz number, increasing up to 2.5 times thicker at lower temperature. Some thickening can also be seen up to a normalised temperature of 0.6 (corresponding to approximately $1220 \mathrm{~K}$ ). In all three cases, there does not appear to be any thickening in the reaction zone.

The response of the thickening factor is dependent on the global Lewis number. To demonstrate this, the thickening factor has also been presented in figure 6 for the lean premixed hydrogen flames from a previous study, [19, which had a global Lewis number of approximately 0.35. A very different response is observed; rather than thickening the flame, the hydrogen flame gets thinner 255 with increased turbulence levels, and in stark contrast to the methane flame, the reaction zone of the hydrogen flame is significantly thinner (the minimum value of $\Theta_{36, \mathrm{H}_{2}}$ is approximately 0.25$)$. Note that this is despite taking the effects of the thermodiffusive instability into account by normalising by the Ka $=1$ flame. Preliminary results in lean premixed dodecane flames suggest that thickening 260 is even more pronounced at high Lewis number, and will be presented in a future paper. It was observed in [19] that turbulence interacts with the hydrogen flame to create regions of high positive curvature, in which enhanced burning was observed as a result of the thermodiffusively unstable nature of the flame; the observed thinning of the reaction zone is a consequence of this enhanced burning, and not observed in the methane flame because of the globally unity Lewis number. There is a slight suggestion that, for low temperatures in the $\mathrm{Ka}=36$ hydrogen case, the thickening factor has increased; it may be the case that the minimum thickness has been reached and turbulence is beginning to have a broadening effect at low temperatures. 


\subsection{Conditional Means of Species Molar Concentrations}

To consider the effect of turbulence on the chemical composition, conditional means of molar concentration as a function of temperature are presented in figures 7 and 8 for the same eight representative species shown in figure 5 at $\mathrm{Ka}=1$ (left) and $\mathrm{Ka}=36$ (right), respectively (note that conditional means 275 for all species are provided as supplementary material). In each plot, the black line shows the conditional mean, the grey area shows one standard deviation either side of the mean, the red line is the unstrained laminar flame with full transport (i.e. the normal temperature-dependent mixture-averaged transport properties), and the blue line is a one-dimensional unstrained laminar flame with 280 unity Lewis number transport (i.e. the normal temperature-dependent thermal diffusivity $\lambda$ was used, but species diffusion coefficients were set to $\mathcal{D}_{i}=\lambda / \rho c_{p}$ ). The dashed black lines in figures 7 and 8 are intermediate profiles from onedimensional unstrained laminar flame calculations where species and thermal diffusion terms have been supplemented by a simple constant turbulent diffusion using a gradient-diffusion hypothesis following

$$
\begin{aligned}
\mathcal{D}_{i} & =\mathcal{D}_{i 0}+\mathcal{D}_{\text {turb }} \\
\lambda & =\lambda_{0}+\rho c_{p} \mathcal{D}_{\text {turb }}
\end{aligned}
$$

where the 0 suffix denotes the usual physical value; note that this means the turbulent Lewis number for all species is one, and the overall Lewis number tends to one in the limit of high turbulent diffusion. The values shown for $\mathcal{D}_{\text {turb }}$ presented are $\{1,3.1,10,31,100$, and 310$\} \times 10^{-6} \mathrm{~m}^{2} / \mathrm{s}$. For comparison, for the reactant mixture at $298 \mathrm{~K}$, the diffusion coefficients are $\mathcal{D}_{\mathrm{CH}_{4}}=2.29, \mathcal{D}_{\mathrm{H}_{2}}=7.9$, $\mathcal{D}_{\mathrm{H}}=12.2$, and $\lambda / \rho c_{p}=2.21\left(\right.$ all $\left.\times 10^{-6} \mathrm{~m}^{2} / \mathrm{s}\right)$. The actual properties of turbulence change across the flame due to expansion with increasing temperature, so such a simple model is not expected to be appropriate. A comprehensive study of turbulence models is beyond the scope of this paper, but this simple approach 295 can be used to gain some preliminary insight into what would be required of a turbulent flame model to capture changes to chemical composition. Naturally, at $\mathcal{D}_{\text {turb }}=0$, the normal full-transport diffusion coefficients are recovered, and the Lewis number will tend to unity for large $\mathcal{D}_{\text {turb }}$. It should be noted that for this simple turbulence model the resulting flame thickness is much broader (dimensional analysis suggests it should scale with $\mathcal{D}_{\text {turb }}^{1 / 2}$ ), and as a result there is a shift in the balance between chemical and diffusive timescales such that the limiting chemical distribution is not the same as the unity Lewis number profile calculated above. However, for the range of values of $\mathcal{D}_{\text {turb }}$ presented here, the general trend in chemical composition is from the full transport profile to the unity Lewis number profile.

The methane profiles for $\mathrm{Ka}=1$ and 36 depicted in figure 7(a,b) (representative of reactants) show almost no variation; the three-dimensional simulations, the unstrained laminar flame, and the unity Lewis number profile all have the same distribution. At $\mathrm{Ka}=1$, carbon dioxide, figure 7(c), follows the unstrained

310 laminar flame closely with little variation. At $\mathrm{Ka}=36$, figure 7(d), the mean distribution has moved towards the unity Lewis number profile, and is close to 
the $\mathcal{D}_{\text {turb }}=10^{-4}$ profile; also, the variation has increased. This $\mathrm{CO}_{2}$ profile is representative of the $\mathrm{H}_{2} \mathrm{O}$ profile in the sense that there is shift at $\mathrm{Ka}=36$ towards the unity Lewis number profile; however, the $\mathrm{H}_{2} \mathrm{O}$ unity Lewis number profile is lower than the full-transport profile, rather than higher. Similar behaviour is observed in the ethane profiles, figure 7(e,f), which is representative of the stable intermediates; the $\mathrm{Ka}=1$ profile is close to the unstrained laminar flame, while at low temperatures, the $\mathrm{Ka}=36$ profile has moved towards the unity Lewis number distribution with low variation, but at intermediate temper-

320 atures, the conditional mean is marginally outside the range of one-dimensional unstrained laminar flame profiles. Note in particular that there is typically a higher molar concentration of these intermediate species in the preheat zone (see supplementary material) and that, because the conditional means are presented as a function of temperature, the increase is implicitly compounded by the thermal thickening presented in figure 6. $\mathrm{HO}_{2}$ profiles are shown in figure $7(\mathrm{~g}, \mathrm{~h})$, where, at $\mathrm{Ka}=36$, there is a much higher variation about the mean, but the shift from the unstrained laminar profile to the unity Lewis number profile is observed over a broad range of temperatures; the mean is close to the $\mathcal{D}_{\text {turb }}=10^{-5}$ profile. The low-temperature radical $\mathrm{CH}_{3} \mathrm{O}$ is shown in fig330 ure $8(\mathrm{a}, \mathrm{b})$ and, $\mathrm{Ka}=36$, presents the largest variation about the mean, has a pronounced shift from the unstrained laminar flame to the unity Lewis number profile, and is again close to the $\mathcal{D}_{\text {turb }}=10^{-5}$ profile. It is interesting to note that the inconsistency between the profiles at high $\mathcal{D}_{\text {turb }}$ and Le $=1$ is apparent in the species with low-temperature activity. The response of molecular hydro-

335 gen is particularly interesting because of the significant difference between the unstrained laminar flame and the unity Lewis number profile. $\mathrm{H}_{2}$ is highly diffusive, and so with full transport, the highest molar concentration occurs at low temperatures $(T \approx 400 \mathrm{~K})$. With unity Lewis number transport, the penetration of $\mathrm{H}_{2}$ into the preheat zone is restricted, which is also observed at $\mathrm{Ka}=36$, fig340 ure $8(\mathrm{~d})$, where the profile is between the $\mathcal{D}_{\text {turb }}=3.1 \times 10^{-5}$ and $10^{-5}$ profiles; at higher temperatures, the profile appears to be little affected by turbulence. Atomic hydrogen, while also being highly mobile, does not penetrate the preheat zone due its high reactivity, and is only observed at high temperatures. It is interesting to note that the unity Lewis number profile has a higher peak than

345 the unstrained laminar flame and the turbulent flame profiles; there is effectively a drop in the Lewis number of atomic hydrogen, which means that the profile is narrower in temperature space (note this gives no information about distribution in physical space, but figure 6 shows that the spatial distribution does not change significantly at these high temperatures). The atomic hydrogen profile at $\mathrm{Ka}=36$, figure 8 (f), is close to $\mathcal{D}_{\text {turb }}=3.1 \times 10^{-5}$. Finally, HCO is shown in figure $8(\mathrm{~g}, \mathrm{~h})$ where the response is much less than other species; the effect of turbulence is much lower in the reaction zone than at lower temperatures, but there is still an increase in variation about the mean and a slight shift towards the unity Lewis number profile.

In summary, the conditional means of species show that there is a shift from the unstrained laminar flame towards a unity Lewis number profile, which occurs at low temperatures first. This shift results in higher molar concentrations of 
stable intermediates in the preheat zone, and large variations about the mean for species involved in low-temperature chemistry. The response in the reaction zone is slight compared to in the preheat zone. A particularly interesting profile to consider is molecular hydrogen; it is highly mobile and so presents high concentrations at low temperatures, which are not present in the unity Lewis number profile. As turbulence becomes more important, packets of species are advected together, so the effects of differential diffusion become less important.

365 Similar effects were shown in [16] for fuel mass fraction and in terms of reduced variation in local equivalence ratio. The conditional means presented here show that with increasing $\mathrm{Ka}$, the chemical compositions shift towards that of a unity Lewis number flame, reflecting the increasing importance of turbulent mixing. This is seen first at low temperatures, and then at higher temperatures 370 as the Karlovitz number is increased. Note that since the conditional means are presented as a function of temperature, the shift to unity Lewis number behaviour is compounded by the thickening of the preheat zone presented in section 3.4 .

We argue that this shift in chemical composition is actually the beginning of

375 the transition to distributed burning (see [15, 16, 9] and the references therein for a detailed examination of the transition to distributed burning in supernova flames, lean premixed hydrogen, and larger hydrocarbons, respectively; other relevant studies include [21, 22, 10, 11, 23, 24, 25]). Distributed burning is the limiting behaviour when turbulent mixing dominates species and thermal 380 diffusion; all fluid parcels are advected together, and the associated turbulent diffusion dominates the molecular diffusion (and consequentially any changes in equivalence ratio) in this regime. This results in effectively unity Lewis number transport. In [16] and [9, it was shown that in high Karlovitz number flames, turbulence leads to a reduction in variation in local equivalence ratio, and the fuel mass fraction collapses to a unity Lewis number curve. In Savard et al. [26], an a priori model for effective species Lewis number is proposed based on data from [16, which tends towards unity for high Karlovitz numbers. Srinivasan et al. 27] also observe a tendency towards unity Lewis number behaviour using the one-dimensional LEM approach. This further demonstrates that the phe390 nomenon occurs in methane flames, in slightly different ways depending on the type of chemical species, and that it occurs first at low temperatures, and progresses through the flame as Karlovitz number increases. The Karlovitz numbers of the flames presented in the present paper (maximum of 36 compared with 410 in [16]) are far too low to observe distributed burning, but the onset of the transition can be seen at low temperatures where the influence of turbulence is strongest. Thermal expansion through the flame reduces the influence of turbulent mixing on the flame, which is compounded by the effect of increasing diffusivities of species and heat with increasing temperature.

\section{Diffusion Study}

400

The slices of fuel consumption rate and heat release in figure 2 along with the curvature JPDFs in figure 4 showed that there is a decrease in reaction rates 
in regions of high positive curvature. A possible consequence of this is that the flame surface is 'leaking' intermediate species, i.e. where there is a reduction in reaction rates, there is a corresponding increase in chemical timescale (local drop in $\mathrm{Da}$ and increase in $\mathrm{Ka}$ ) that may allow turbulent mixing to draw partially reacted parcels of fluid into the preheat zone. Natural candidates to consider here, because of their high diffusivities, are atomic and molecular hydrogen.

To investigate these possibilities, a diffusion study has been undertaken whereby additional three-dimensional simulations have been run at $\mathrm{Ka}=36$ ${ }_{410}$ where the diffusion coefficients of selected species have been artificially changed. Specifically, three different variants have been considered: two by setting the diffusion of atomic and molecular hydrogen independently to unity Lewis number, and finally setting all species to unity Lewis number. The three variant cases will be denoted by $\mathrm{Le}_{\mathrm{H}}=1, \mathrm{Le}_{\mathrm{H}_{2}}=1$, and $\mathrm{Le}_{i}=1$, respectively. To save computational expense, the simulations were conducted at a smaller length scale ( $\Lambda=1$ instead of 4$)$, aspect ratio (4 instead of 8 ) and computational resolution (a base grid of $192 \times 192 \times 768$ with no AMR); since the focus of the study is small-scale interaction in the reaction zone, this will not affect the conclusions. A control simulation with full transport was run at this reduced resolution to ${ }_{420}$ match the larger simulations presented in the previous section. An advantage of running at reduced expense with no AMR means that temporal averaging can be performed to obtain better converged statistics.

\subsection{Overview}

To allow for slices to be compared at the same time, a simulation with full transport was run first up to a restart time point. Then the four different configurations were restarted from this time point and run for the same period of time (about $2.6 \mathrm{~ms}$, which corresponds to about $75 \%$ of a flame time scale). Slices of fuel consumption rate along with molecular and atomic hydrogen mass fraction are shown in figures 9 and 10 for the four cases; note that periodicity ${ }_{430}$ has been exploited to stitch together slices at $x=0$ and $y=0$ to show more flame surface, so the slices are two domain widths across and four high. The slice for each field has been normalised by the peak value from the corresponding unstrained laminar flame.

Figure 9 compares the full transport case (top) with the $\mathrm{Le}_{\mathrm{H}_{2}}=1$ case ${ }_{435}$ (bottom). This comparison shows that the fuel consumption and molar concentration of atomic hydrogen appear to be very similar in the two different cases. Importantly, the decrease in reaction rates in regions of high positive curvature can be seen in both cases (as indicated by the white arrows). The profile of molecular hydrogen for the $\mathrm{Le}_{\mathrm{H}_{2}}=1$ case is slightly narrower, presents much 440 less variability along the flame surface, and appears less diffuse in the preheat zone; these are natural consequences of reduced molecular hydrogen diffusion. Other than the profile of $\mathrm{H}_{2}$, setting $\mathrm{Le}_{\mathrm{H}_{2}}=1$ has little effect on the flame response to turbulence. Figure 10 compares the $\mathrm{Le}_{\mathrm{H}}=1$ (top) and $\mathrm{Le}_{i}=1$ (bottom) cases. These two cases again appear to be very similar to each other,

${ }_{445}$ the main difference being the broader profile and higher values of $\mathrm{H}_{2}$ molar concentration in the $\mathrm{Le}_{\mathrm{H}}=1$ due to the higher diffusivity in that case. 
The key point to note is that the two cases in figure 9 are similar to each other, and the two cases in figure 10 are similar to each other, but figures 9 and 10 are quite different. Specifically, the reduced fuel consumption in regions of high positive curvature (as highlighted by the white arrows) can be seen in figure 9 but not in figure 10 . This suggests that the decrease in reaction rates in regions of high positive curvature can be attributed primarily to the diffusion of atomic hydrogen.

\subsection{JPDFs of Flame Speed and Curvature}

Figure 11 shows joint probability density functions of normalised consumptionbased turbulent flame speed and curvature for the four diffusion study cases, similar to the $\Lambda=4$ cases presented in figure 4 note the flame speeds have been normalised by the unstrained laminar flame corresponding to each case. It is interesting to note that the flame speed of the $\mathrm{Le}_{\mathrm{H}_{2}}=1$ case is about $10 \%$ faster 460 than the other three cases, and the $\mathrm{Le}_{\mathrm{H}_{2}}=1$ and $\mathrm{Le}_{i}=1$ cases are about $7 \%$ thinner (see table 21. The full transport case presents the same negative correlation observed at $\Lambda=4$, but with less variation. When $\mathrm{Le}_{\mathrm{H}_{2}}=1$, the peak has a normalised flame speed that is close to one, but slightly negative curvature, and there is an increased variation. For $\mathrm{Le}_{\mathrm{H}}=1$, the JPDF is flatter, has a peak with a normalised flame speed about $5 \%$ faster than the corresponding unstrained laminar flame, and has moved to higher values of curvature; there is a greater variation at negative curvature than at positive curvature. The unity Le case also presents a flatter JPDF, with a peak close to a normalised flame speed of one and a curvature of zero; there is less variation at negative curva-

470 ture than in the $\mathrm{Le}_{\mathrm{H}}=1$ case. Again, just by setting the diffusion of atomic hydrogen to unity Lewis number, the flame response to turbulence is very close to that of a flame where every species has unity Lewis number.

\subsection{Thickening Factor}

To consider whether these reduced reaction rates are related to the thickening

475 of the preheat zone, figure 12 presents the thickening factor for the four different configurations used in the diffusion study, along with the $\Lambda=4$ flames from the previous sections. Note that these simulations were run from scratch (without restarting from a common time point) so that the evolution of the preheat zone in each case develops independently. The first point to note is that there is little, if any difference, between the full transport simulations at $\Lambda=4$ and 1 , supporting the use of smaller scale simulations. The second point to note is that with unity Lewis number atomic hydrogen diffusion (in both the $\mathrm{Le}_{\mathrm{H}}=1$ and $\mathrm{Le}_{i}=1$ cases), the reaction zone is actually thinner than the full transport simulations (and the $\mathrm{Le}_{\mathrm{H}_{2}}=1$ case). There is a slight decrease in the thickening

485 at low temperatures in the $\mathrm{Le}_{\mathrm{H}}=1$ case. We argue that this is not sufficient to be able to conclude that the reduced reaction rates in regions of high positive curvature are related to the thickening of the preheat zone, especially since the $\mathrm{Le}_{i}=1$ appears to be thickened as much as the full transport case. 


\subsection{Conditional Means of Species Molar Concentrations}

Figures 13 and 14 present conditional means of molar concentration for four of the species considered previously for all four cases in the diffusion study; specifically, $\mathrm{CO}_{2}, \mathrm{HO}_{2}, \mathrm{CH}_{3} \mathrm{O}$ and $\mathrm{H}_{2}$, the rest can be found in the supplementary material. The conditional means of $\mathrm{CO}_{2}$ for the full transport and $\mathrm{Le}_{\mathrm{H}_{2}}=1$ cases are almost indistinguishable; for the $\mathrm{Le}_{\mathrm{H}}=1$ case, there is a slight shift towards the one-dimensional unity Lewis number profile, but in the $\mathrm{Le}_{i}=1$ case, the conditional mean overlies the unity Lewis number profile. This response is reflected in the other stable intermediates (see supplementary material). A similar response is also observed in $\mathrm{HO}_{2}$ and $\mathrm{CH}_{3} \mathrm{O}$, but the shift in the $\mathrm{Le}_{\mathrm{H}}=1$ case is much more significant, and the $\mathrm{Le}_{i}=1$ case doesn't quite line up 500 with the unity Lewis number unstrained laminar profile. This response can also be found in the other species that display low-temperature activity (again, see supplementary material). Interestingly, the response of the conditional mean for $\mathrm{H}_{2}$ is different, in that the $\mathrm{Le}_{\mathrm{H}}=1$ case is similar to the full transport case. Naturally, the response in the $\mathrm{Le}_{\mathrm{H}_{2}}=1$ case is a shift towards the unity Lewis number profile, and the $\mathrm{Le}_{i}=1$ cases lines up closely. The response in the high-temperature species is similar to the stable intermediates in as much as the full transport case and $\mathrm{Le}_{\mathrm{H}_{2}}=1$ case are similar, there is a slight shift towards unity Lewis number in the $\mathrm{Le}_{\mathrm{H}}=1$ case, and the $\mathrm{Le}_{i}=1$ case matches the unity Lewis number profile closely (see supplementary material).

\section{Conclusions}

Three-dimensional direct numerical simulations using the GRIMech 3.0 detailed chemical mechanism have been presented for lean premixed methane flames in the thin reaction zone, with Karlovitz numbers ranging from 1 to 36 , and an integral length approximately four times the thermal thickness of

515 the unstrained laminar flame. An increase in flame surface area was observed with increasing Karlovitz number, and a decrease in the size of local structures. The global flame speed increased with Ka up to a factor of ten, which can be attributed to the increased flame surface area, because only small (around 5\%) increases in local consumption-based speeds were observed. Despite a global

${ }_{520}$ Lewis number (i.e. the Lewis number of the deficient reactant) close to unity, variation along the flame surface was observed at high Ka. A slight negative correlation of local flame speed to mean flame curvature was found at high Ka.

With increasing Ka, thickening of the flame was observed at low temperatures, with parts of the preheat zone found to be over twice as thick at $\mathrm{Ka}=36$ than at $\mathrm{Ka}=1$; the thickness of the reaction zone was found to be unaffected at these Karlovitz numbers. With increasing Ka, a transition was observed in the preheat zone towards the composition found in an unstrained steady flame with unity Lewis number transport. While the reactants were largely unaffected, products and stable intermediate species typically had higher molar concen-

${ }_{530}$ tration in the preheat zone. High-temperature radicals (including $\mathrm{H}$ ) tended towards the unity Lewis number profiles, and the species with low-temperature 
activity showed a large variability in conditional mean and a strong sensitivity to curvature.

A simple model for turbulent species diffusion was shown to reproduce the observed shifts toward unity Lewis number behavior in many species. While this simple construct is not intended for modelling turbulent premixed flames, it does illustrate that turbulence produces a similar response in chemical composition to enhanced diffusion, and suggests a possible approach that can be incorporated into flamelet-based models. Much more work will be required to 540 consolidate this observation into a predictive model.

Finally, the role of molecular and atomic hydrogen diffusion was examined using additional three-dimensional simulations with artificial diffusion models. It was shown that the variability along the flame surface for the high Ka cases can be attributed predominately to the diffusion of atomic hydrogen.

In summary, we argue that the leading-order response of the flame can be attributed to the global Lewis number, while second-order effects observed at the flame surface are attributed to diffusion of atomic hydrogen. Specifically, for unity global Lewis number methane flames, there is thickening of the preheat zone at low temperatures, unlike the low global Lewis number hydrogen flames, ${ }_{550}$ which are found to be thinner for all temperatures. Preliminary results in high global Lewis number dodecane flames suggest that the thickening is more pronounced, and will be explored in a future paper. In the case of the present lean methane flames, a slight decrease in reaction reaction rates was observed in regions of high positive curvature, which was attributed to atomic hydrogen

555 diffusion. Diffusion of atomic hydrogen has also been found to be responsible for the decorrelation between fuel consumption and heat release reported in [19] for lean premixed hydrogen flames, and will also be the subject of a future paper.

\section{Acknowledgements}

AJA would like to thank Ed Richardson for many useful discussions. JBB and MSD were supported by the DOE Applied Mathematics Research Program of the DOE Office of Advanced Scientific Computing Research under the U.S. Department of Energy Contract No. DE-AC02-05CH11231.

\section{References}

\section{References}

[1] N. Peters, P. Terhoeven, J. H. Chen, T. Echekki, Statistics of flame displacement speeds from computations of 2-D unsteady methane-air flames, Symposium (International) on Combustion 27 (1) (1998) 833 - 839.

[2] J. H. Chen, H. G. Im, Correlation of flame speed with stretch in turbulent premixed methane/air flames, Symposium (International) on Combustion 27 (1) (1998) $819-826$. 
[3] T. Echekki, J. H. Chen, Analysis of the contribution of curvature to premixed flame propagation, Combustion and Flame 118 (12) (1999) 308 311.

[13] G. P. Smith, D. M. Golden, M. Frenklach, N. W. Moriarty, B. Eiteneer, M. Goldenberg, C. T. Bowman, R. K. Hanson, S. Song, W. C. Gardiner Jr., pocket formation in lean premixed methane-air flames with implications to turbulent combustion, Combustion and Flame 116 (12) (1999) 15 - 48.

[5] J. B. Bell, M. S. Day, J. F. Grcar, Numerical simulation of premixed turbulent methane combustion, Proc. Combust. Inst. 29 (2002) 1987-1993.

[6] J. B. Bell, M. S. Day, I. G. Shepherd, M. Johnson, R. K. Cheng, J. F. Grcar, V. E. Beckner, M. J. Lijewski, Numerical simulation of a laboratory-scale turbulent V-flame, Proc. Natl. Acad. Sci. USA 102 (29) (2005) 1000610011.

[7] J. B. Bell, M. S. Day, J. F. Grcar, M. J. Lijewski, J. F. Driscoll, S. F. Filatyev, Numerical simulation of a laboratory-scale turbulent slot flame, Proc. Combust. Inst. 31 (2007) 1299-1307.

[8] R. Sankaran, E. R. Hawkes, J. H. Chen, T. Lu, , C. K. Law, Structure of a spatially developing turbulent lean methane-air bunsen flame, Proceedings of the Combustion Institute 31 (1) (2007) 1291 - 1298.

[9] A. J. Aspden, M. S. Day, J. B. Bell, Lewis number effects in distributed flames, Proceedings of the Combustion Institute 33 (1) (2011) 1473 - 1480.

[10] H. Carlsson, R. Yu, X.-S. Bai, Direct numerical simulation of lean premixed $\mathrm{CH}_{4}$ /air and $\mathrm{H}_{2}$ /air flames at high Karlovitz numbers, International Journal of Hydrogen Energy 39 (35) (2014) 20216-20232.

[11] H. Carlsson, R. Yu, X.-S. Bai, Flame structure analysis for categorization of lean premixed $\mathrm{CH}_{4}$ /air and $\mathrm{H}_{2}$ /air flames at high Karlovitz numbers: Direct numerical simulation studies, Proceedings of the Combustion Institute 35 (2) (2015) 1425-1432.

[12] M. Frenklach, H. Wang, M. Goldenberg, G. P. Smith, D. M. Golden, C. T. Bowman, R. K. Hanson, W. C. Gardiner, V. Lissianski, GRI-Mechan optimized detailed chemical reaction mechanism for methane combustion, Tech. Rep. GRI-95/0058, Gas Research Institute, http://www.me. berkeley.edu/gri_mech/(1995).

[4] J. H. Chen, T. Echekki, W. Kollmann, The mechanism of two-dimensional 
[15] A. J. Aspden, J. B. Bell, M. S. Day, S. E. Woosley, M. Zingale, TurbulenceFlame Interactions in Type Ia Supernovae, The Astrophysical Journal 689 (2008a) 1173-1185.

[16] A. J. Aspden, M. S. Day, J. B. Bell, Turbulence-flame interactions in lean premixed hydrogen: transition to the distributed burning regime, JFM 680 (2011) 287-320.

[17] M. S. Day, J. B. Bell, Numerical simulation of laminar reacting flows with complex chemistry, Combust. Theory Modelling 4 (2000) 535-556.

[18] M. S. Day, J. B. Bell, P.-T. Bremer, V. Pascucci, V. Beckner, M. J. Lijewski, Turbulence effects on cellular burning structures in lean premixed hydrogen flames, Combustion and Flame 156 (5) (2009) 1035 - 1045.

[19] A. J. Aspden, M. S. Day, J. B. Bell, Turbulence-chemistry interaction in lean premixed hydrogen combustion, Proceedings of the Combustion Institute 35 (2) (2015) 1321 - 1329.

[20] A. J. Aspden, M. S. Day, J. B. Bell, Characterization of low lewis number flames, Proceedings of the Combustion Institute 33 (1) (2011) 1463-1471.

[21] A. Y. Poludnenko, E. S. Oran, The interaction of high-speed turbulence with flames: Global properties and internal flame structure, Combustion and Flame 157 (5) (2010) 995-1011.

[22] A. Y. Poludnenko, E. S. Oran, The interaction of high-speed turbulence with flames: Turbulent flame speed, Combustion and Flame 158 (2) (2011) 301-326.

[23] B. Savard, G. Blanquart, Broken reaction zone and differential diffusion effects in high Karlovitz $\mathrm{n}-\mathrm{C}_{7} \mathrm{H}_{16}$ premixed turbulent flames, Combustion and Flame 162 (5) (2015) 2020-2033.

[24] B. Savard, B. Bobbitt, G. Blanquart, Structure of a high Karlovitz n- $\mathrm{C}_{7} \mathrm{H}_{16}$ premixed turbulent flame, Proceedings of the Combustion Institute 35 (2) (2015) 1377-1384.

[25] S. Lapointe, B. Savard, G. Blanquart, Differential diffusion effects, distributed burning, and local extinctions in high Karlovitz premixed flames, Combustion and Flame 162 (9) (2015) 3341-3355.

[26] B. Savard, G. Blanquart, An a priori model for the effective species Lewis numbers in premixed turbulent flames, Combustion and Flame 161 (6) (2014) 1547-1557.

[27] S. Srinivasan, S. Menon, Linear eddy mixing model studies of high Karlovitz number turbulent premixed flames, Flow, Turbulence and Combustion 93 (2) (2014) 189-219. 
50

51

\begin{tabular}{l|c|c|c|c} 
Case & $\mathrm{A}$ & $\mathrm{B}$ & $\mathrm{C}$ & $\mathrm{D}$ \\
\hline \hline$u[\mathrm{~m} / \mathrm{s}]$ & 0.299 & 0.754 & 1.57 & 2.27 \\
$u / s_{F}$ & 1.59 & 4.00 & 8.32 & 17.3 \\
$\mathrm{Ka}$ & 1.0 & 4.0 & 12.0 & 36.1 \\
$\mathrm{Da}$ & 2.52 & 1.0 & 0.481 & 0.231
\end{tabular}

Table 1: Simulation properties.

\begin{tabular}{l|c|c|c|c} 
Case & control & $\mathrm{Le}_{\mathrm{H}_{2}}=1$ & $\mathrm{Le}_{\mathrm{H}}=1$ & $\mathrm{Le}_{i}=1$ \\
\hline \hline$s_{F}[\mathrm{~m} / \mathrm{s}]$ & 0.188 & 0.206 & 0.181 & 0.186 \\
$l_{F}[\mu \mathrm{m}]$ & 662 & 610 & 644 & 616
\end{tabular}

Table 2: Diffusion study flame properties. 


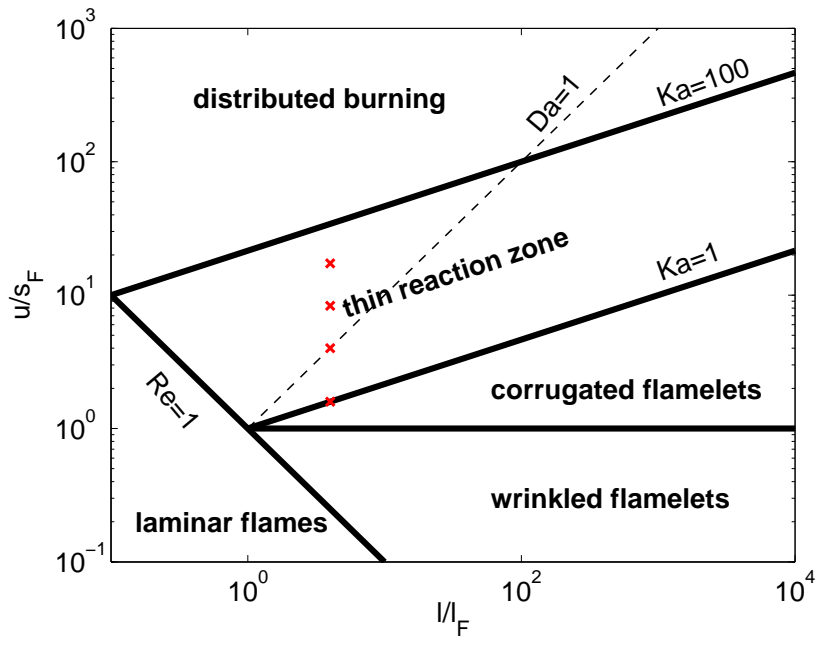

Figure 1: Turbulent premixed regime diagram. The four simulations at $\Lambda=4$ are denoted by red crosses. 


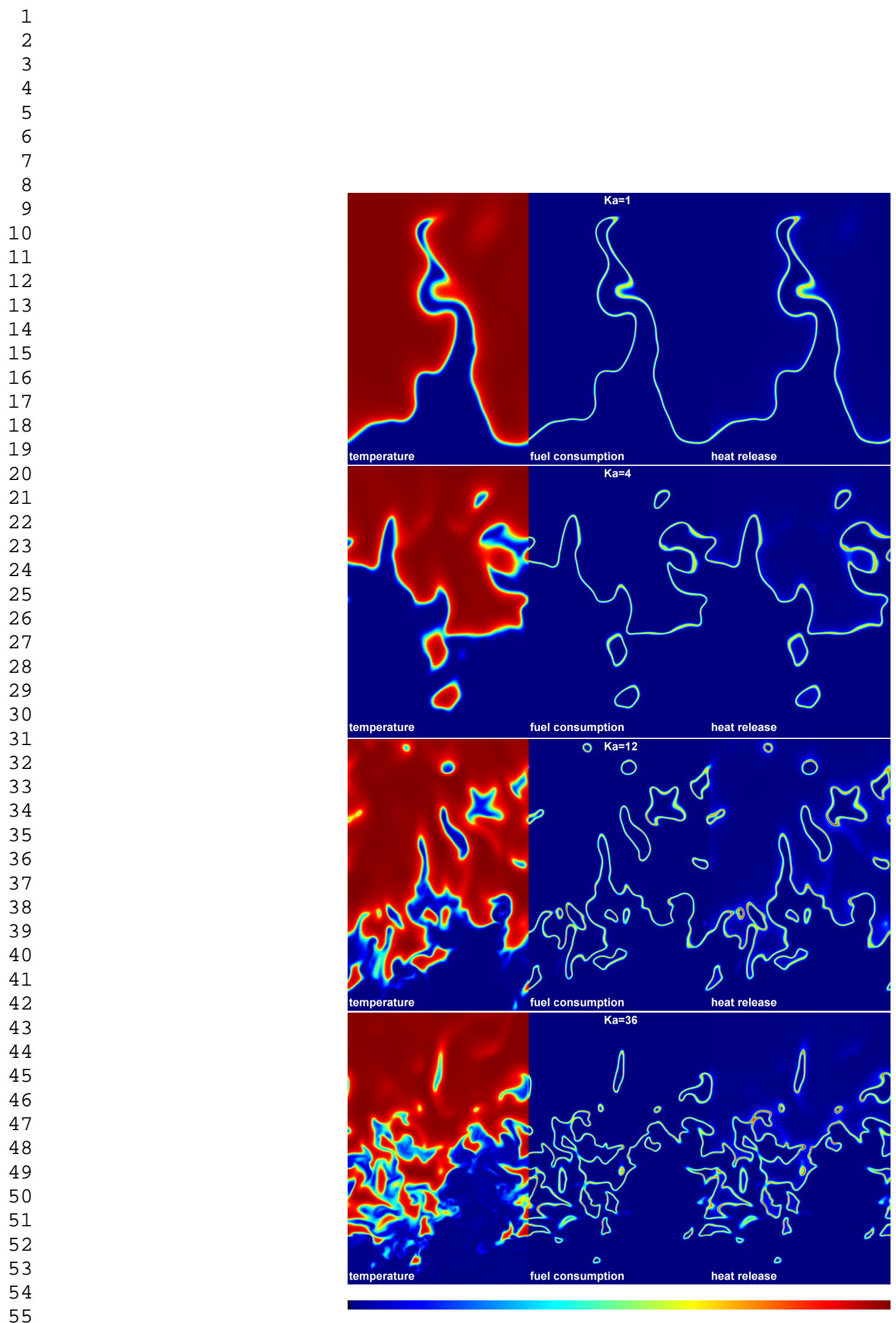

Figure 2: Representative two-dimensional slices through the three-dimensional simulations at $\Lambda=4$. Top-to-bottom are $\mathrm{Ka}=1,4,12$ and 36, respectively. The panels left-to-right are temperature, fuel consumption rate and heat release, respectively. The entire domain width is shown, but only 1.5 domain widths in the vertical direction. 

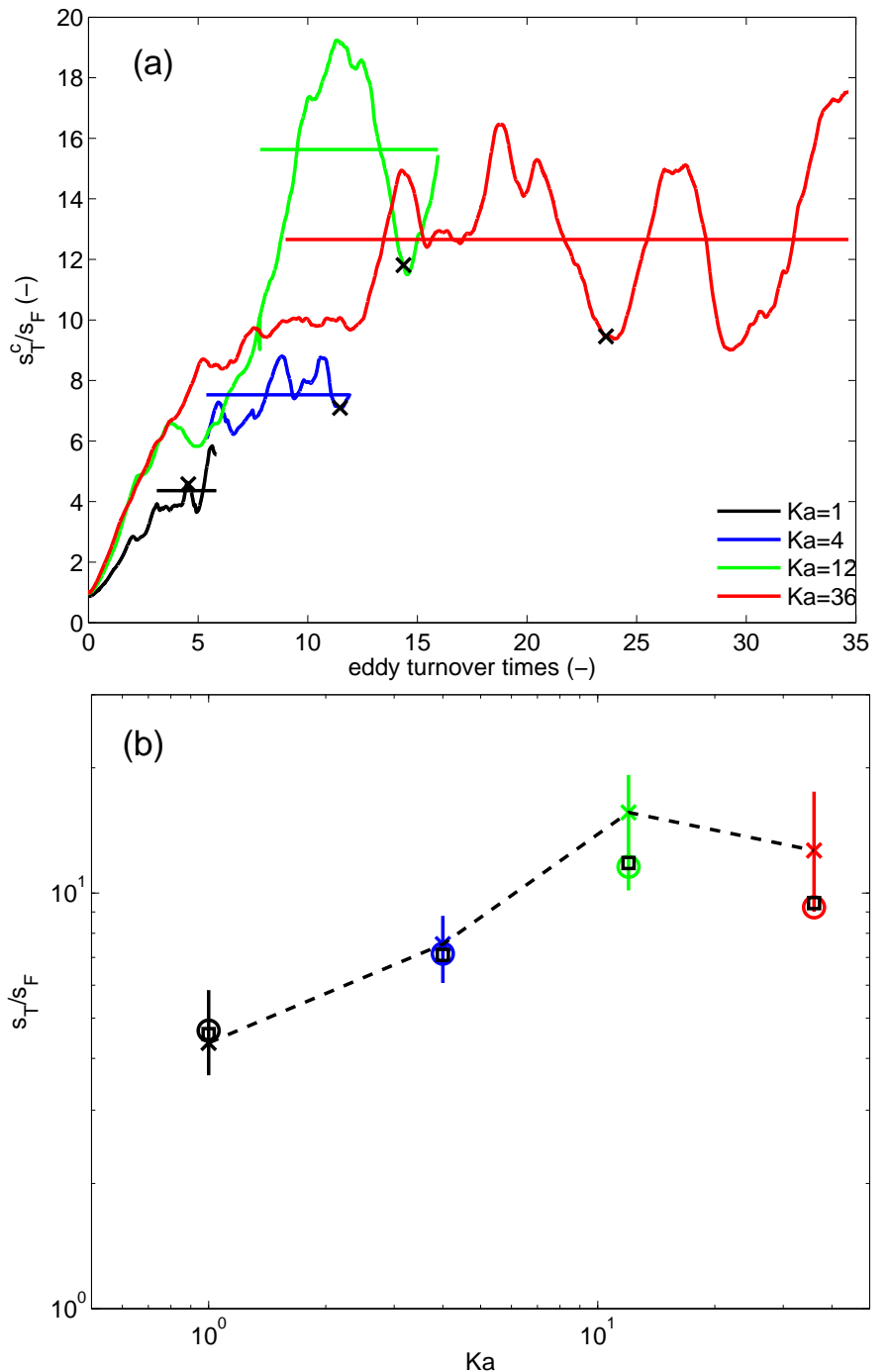

Figure 3: (a) Consumption-based turbulent flame speed as a function of eddy turnover time; crosses denote the time at which statistics were taken, and the horizontal line denotes a nominal average taken after the flame has become established and the period over which it was evaluated. (b) Consumption-based turbulent flame speed as a function of Ka; crosses denote the same average as (a), the vertical line denotes the spread of values attained during the averaging period, circles denote the time at which statistics were taken, which is compared with the normalised flame surface area (based on the isotherm corresponding to peak heat release) denoted by squares. 

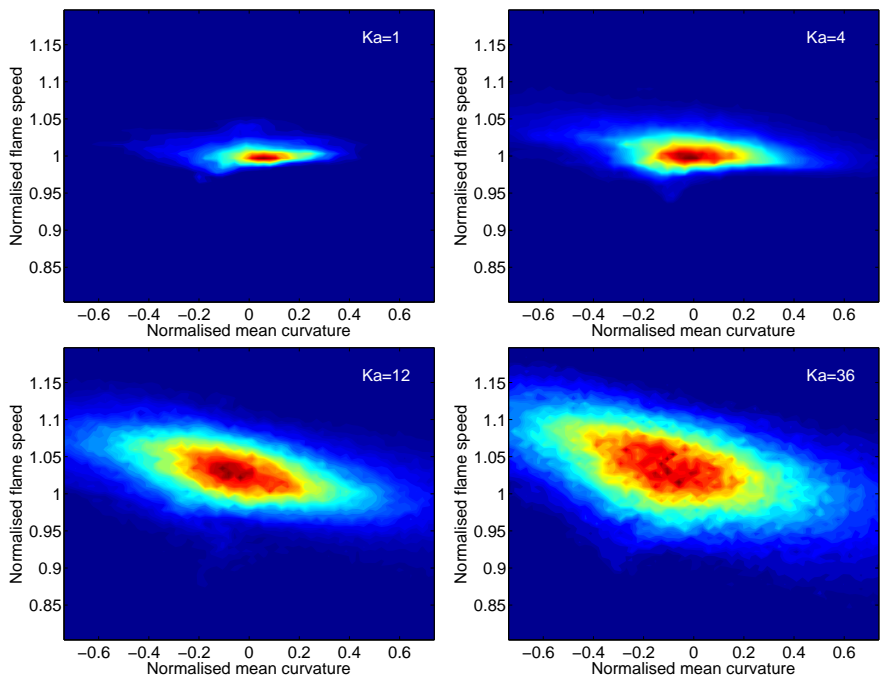

Figure 4: Joint probability density functions of normalised turbulent flame speed $s_{T}^{c} / s_{F}$ and normalised mean curvature $M l_{F}$ for the four cases at $\Lambda=4$. At Ka $=1$, the JPDF is essentially flat, indicating little variation along the flame surface. With increasing Ka, a negative correlation becomes apparent; there is a decrease in the reaction rates at high positive curvature. 

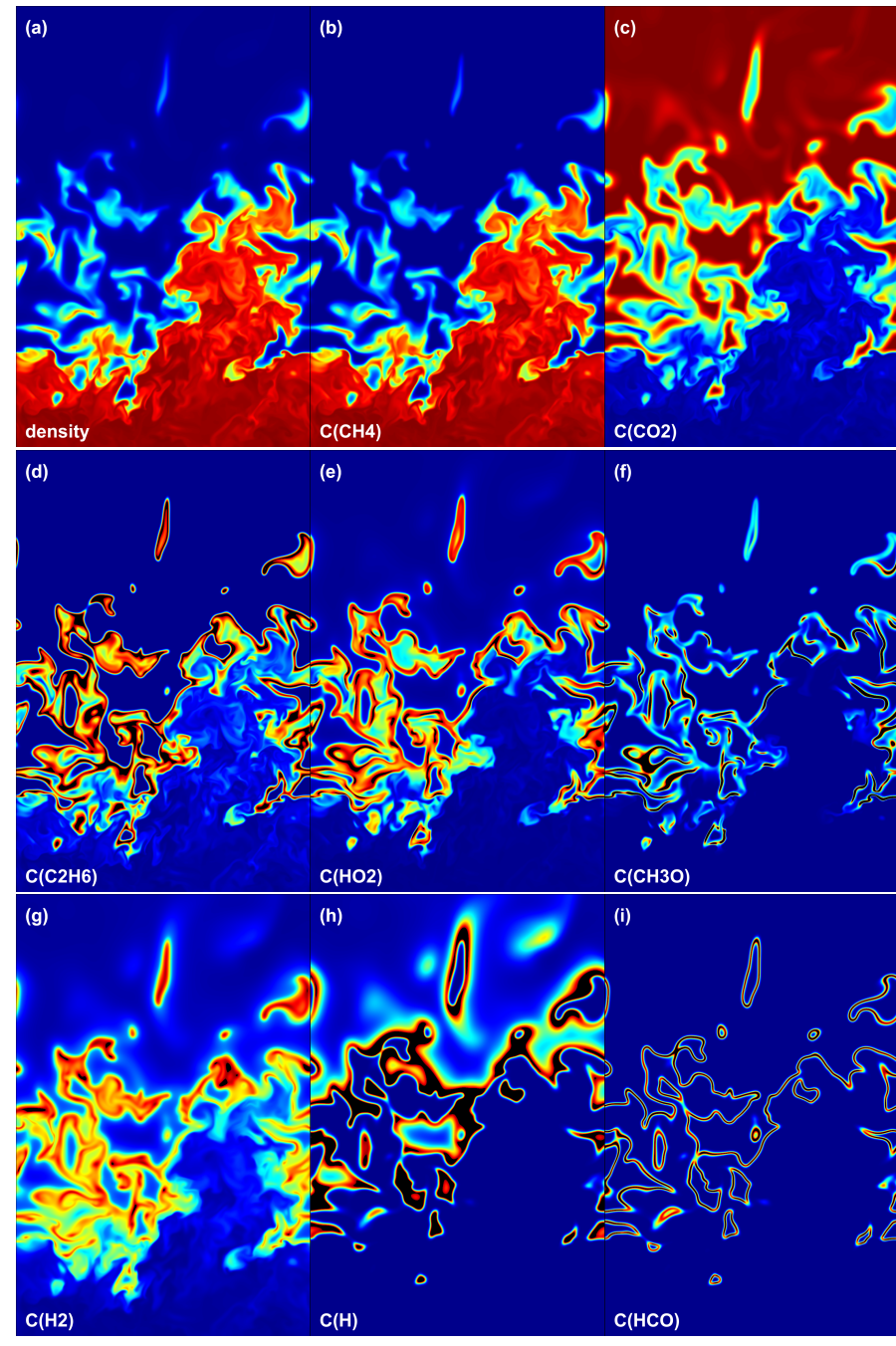

Figure 5: More representative slices through the $\Lambda=4 \mathrm{Ka}=36$ case, showing density and molar concentrations. Species have been chosen as representative of a class of species, or because they are particularly interesting. $\mathrm{CH}_{4}$ is representative of the reactants, $\mathrm{CO}_{2}$ of the products, $\mathrm{C}_{2} \mathrm{H}_{6}$ of stable intermediates, $\mathrm{HO}_{2}$ and $\mathrm{CH}_{3} \mathrm{O}$ of species active at low temperature, and $\mathrm{HCO}$ of high-temperature radicals, $\mathrm{H}_{2}$ and $\mathrm{H}$ are interesting due their high diffusivity. 


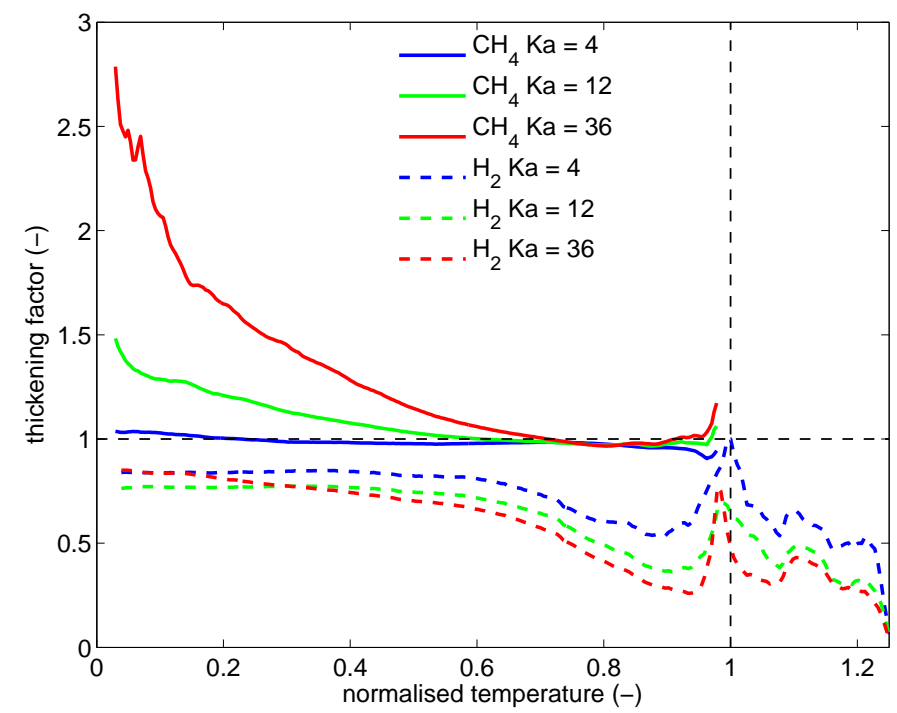

Figure 6: Thickening factor $\Theta(T)$ (defined according to equation 2 for the three higher Ka cases at $\Lambda=4$ (solid lines). Note that there is increasing thickening of the preheat zone with increasing Ka, but the reaction zone thickness is not affected at these Karlovitz numbers. The dashed lines denote the matching hydrogen flames from [19, which show different behaviour; the hydrogen flames become thinner, especially in the reaction zone, as a consequence of the thermodiffusive instability of these low Lewis number flames. 

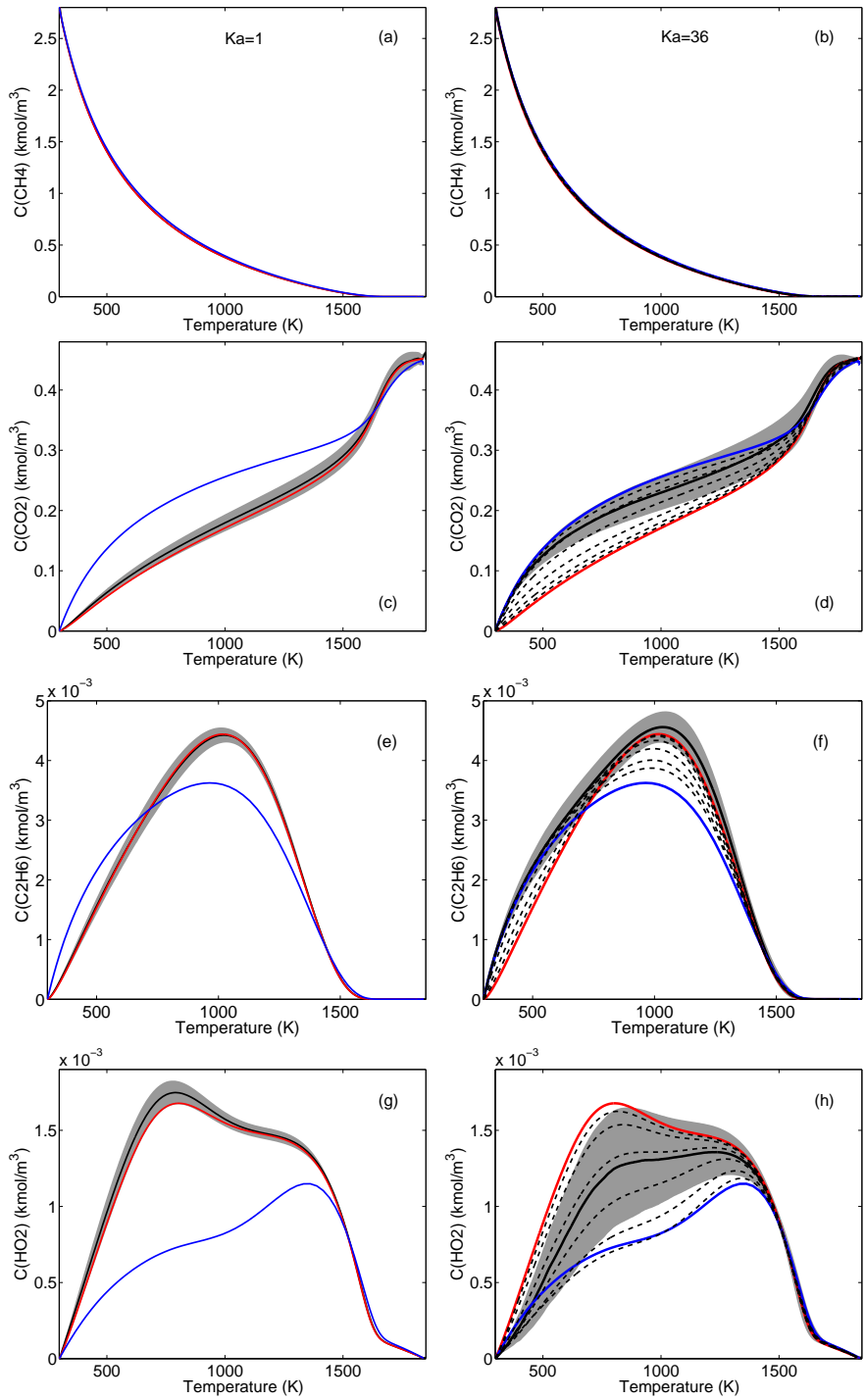

Figure 7: Conditional means of molar concentration (part 1) from the $\Lambda=4$ simulations at $\mathrm{Ka}=1$ (left) and $\mathrm{Ka}=36$ (right). The black line denotes the conditional mean, the grey area shows one standard deviation about the mean, the red line is the corresponding unstrained laminar flame, and the blue line is the profile from a one-dimensional unstrained laminar flame at unity Lewis number. The species shown here and in figure 8 correspond to the same species shown as slices in figure 5 Note how the $\mathrm{Ka}=1$ flame is close to the unstrained laminar flame, that there is a shift towards the unity Lewis number profile at low temperatures in the $\mathrm{Ka}=36$ case, but the conditional mean in the reaction zone is largely unaffected. 

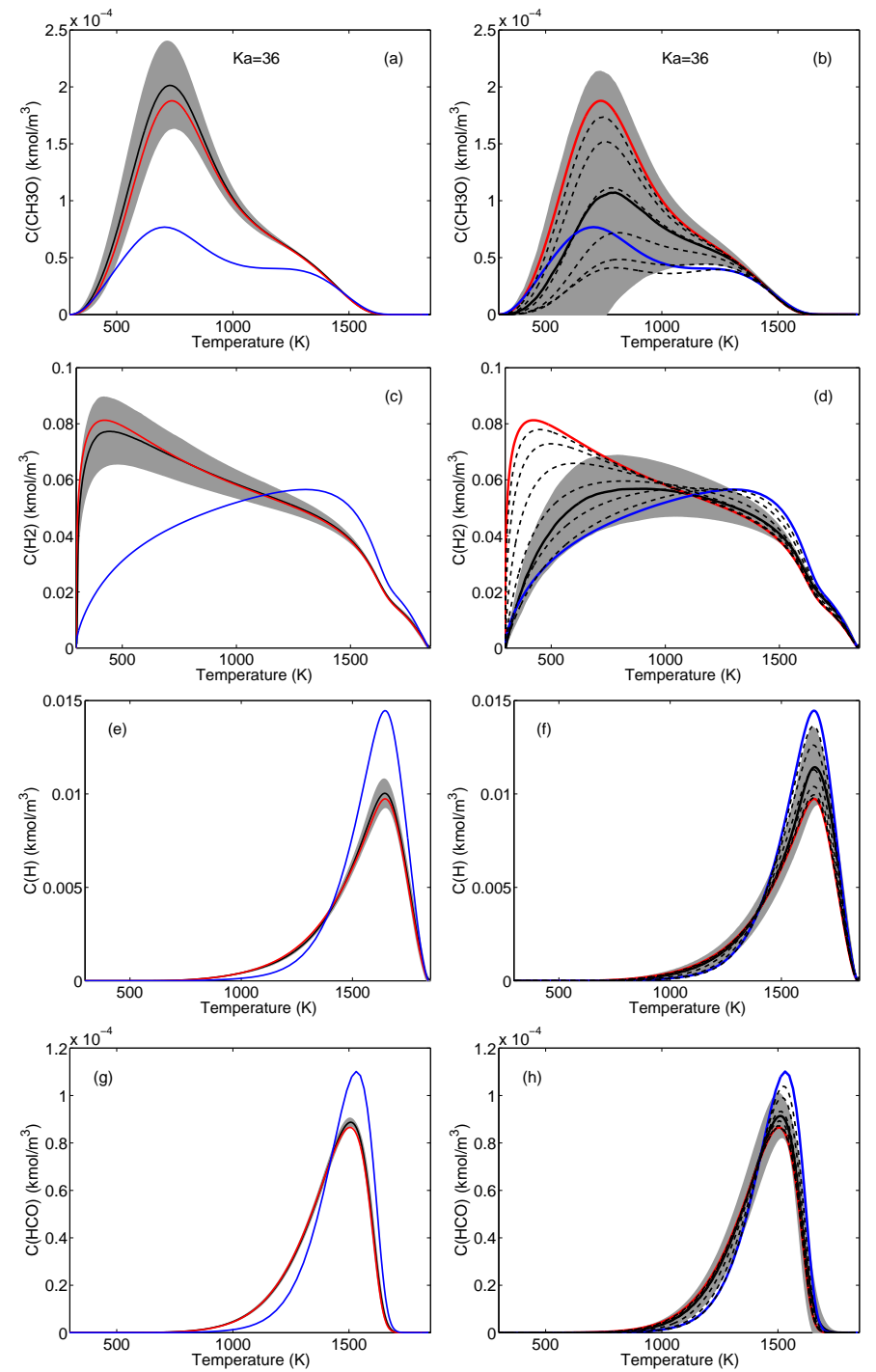

Figure 8: Conditional means of molar concentration (part 2); see the caption of figure 7 for details. 


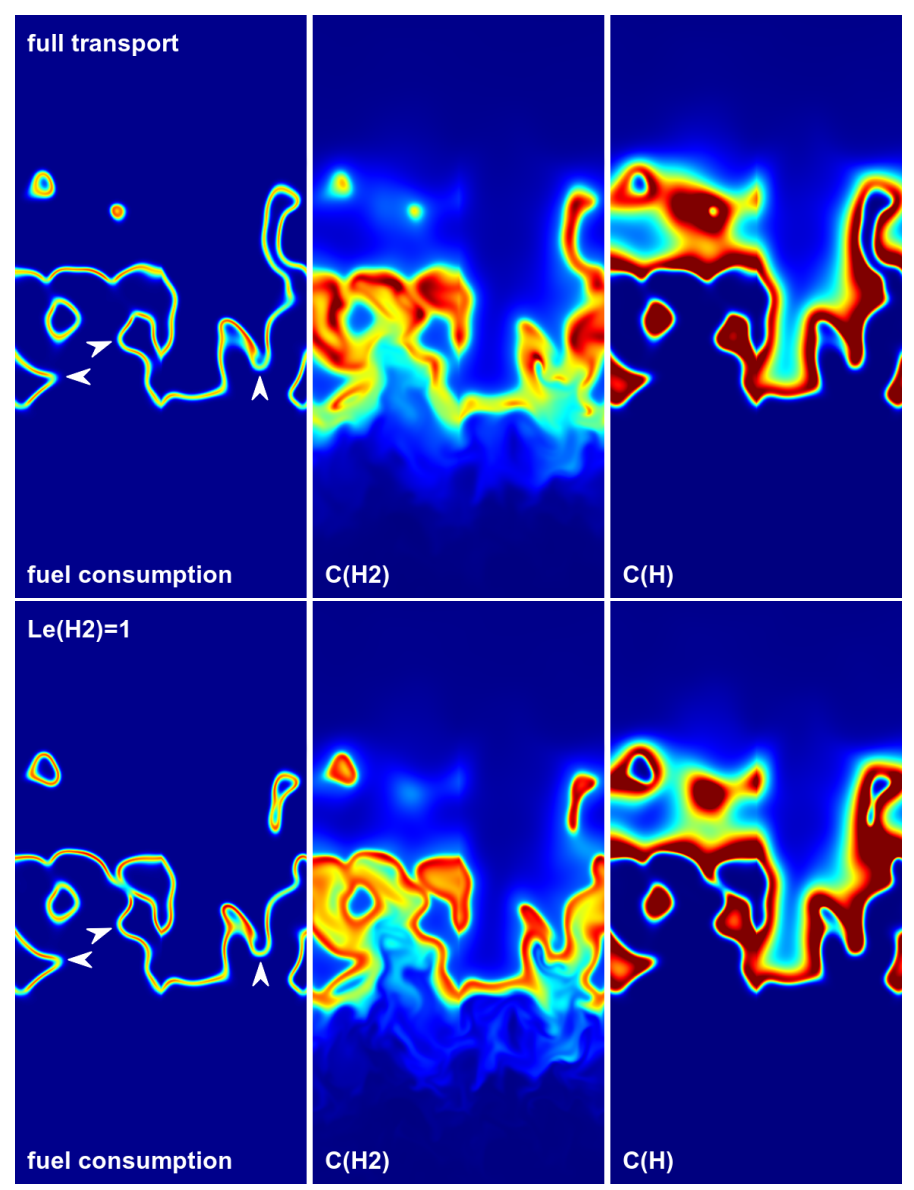

Figure 9: Two-dimensional slices through the three-dimensional data from the diffusion study cases at $\Lambda=1$ (part 1). Note that periodicity has been exploited to stitch together the $x=0$ and $y=0$ slices to show more flame surface. The panels left-to-right are fuel consumption rate, molecular hydrogen mass fraction, and atomic hydrogen mass fraction. Each field has been normalised by the corresponding unstrained laminar flame value. The top panels are for the full transport simulation, and the bottom panels are from the $\mathrm{Le}_{\mathrm{H}_{2}}=1$ case. Note how these two cases are similar to each other (apart from the narrower $\mathrm{H}_{2}$ profile in the bottom panel as a consequence of reduced diffusion), but different from those of figure 10 in particular, the reduced reaction rates in regions of high positive curvature are present in both cases (as highlighted by the white arrows). 


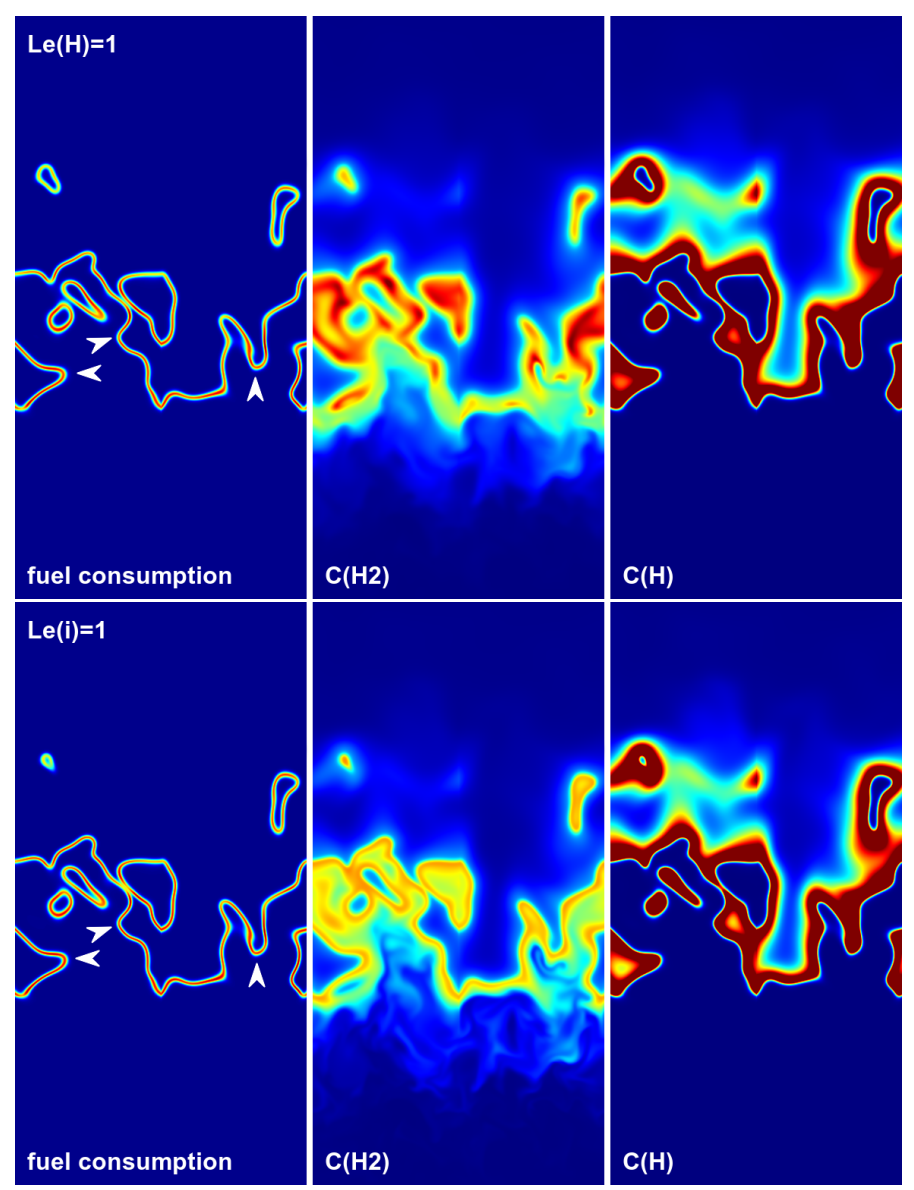

Figure 10: Two-dimensional slices through the three-dimensional data from the diffusion study cases at $\Lambda=1$ (part 2); see the caption of figure 9 for details. The top panels are from the $\mathrm{Le}_{\mathrm{H}}=1$ case, and the bottom panels are from the $\mathrm{Le}_{i}=1$ case. Again, note how these two cases are similar to each other (apart from the narrower $\mathrm{H}_{2}$ profile in the bottom panel as a consequence of reduced diffusion), but different from those of figure 9 in particular, the reduced reaction rates in regions of high positive curvature are no longer present in either case. 
Figure 11: JPDFs of consumption based flame speed and mean curvature for the diffusion study cases. Note in particular how setting just atomic hydrogen to unity Lewis number results in a flame response similar to a flame with unity Lewis number transport for all species. 


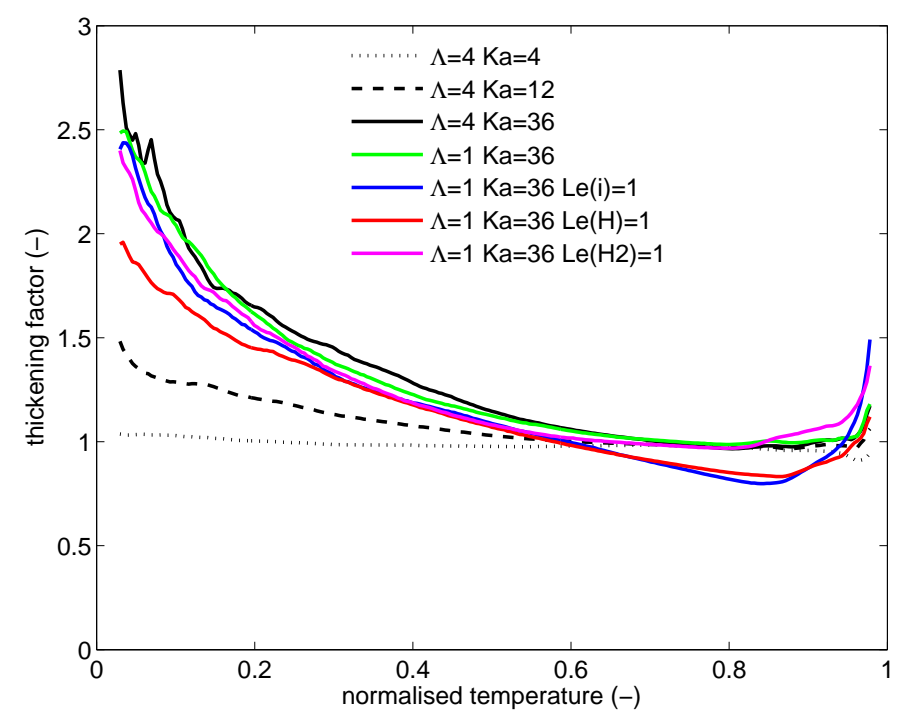

Figure 12: Thickening factor $\Theta(T)$ for the diffusion study cases at $\Lambda=1$, compared with the $\Lambda=4$ cases. Note how cases with full transport at $\Lambda=1$ and $\Lambda=4$ have similar profiles. The cases with reduced atomic hydrogen diffusion are actually thinner in the reaction zone. All of the cases at $\mathrm{Ka}=36$ show significant thickening at low temperatures. 

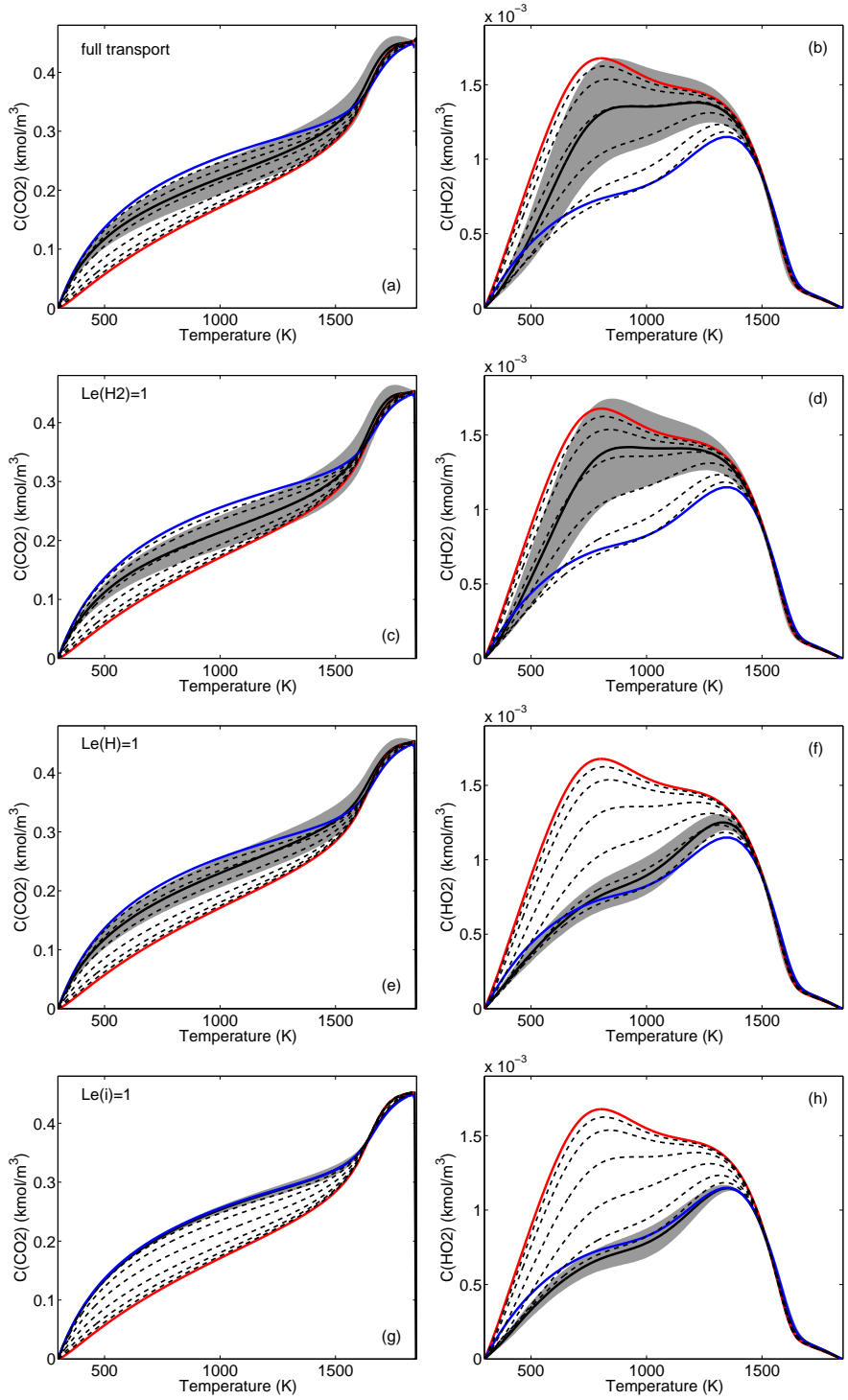

Figure 13: Conditional means of molar concentration (part 1) from the diffusion study cases for $\mathrm{CO}_{2}$ (left) and $\mathrm{HO}_{2}$ (right); top-to-bottom are with full transport, $\mathrm{Le}_{\mathrm{H}_{2}}=1$, Le $\mathrm{H}=1$, and $\mathrm{Le}_{i}=1$, respectively. The black line denotes the conditional mean, the grey area shows one standard deviation about the mean, the red line is the corresponding unstrained laminar flame, and the blue line is the profile from a one-dimensional unstrained laminar flame at unity Lewis number. 

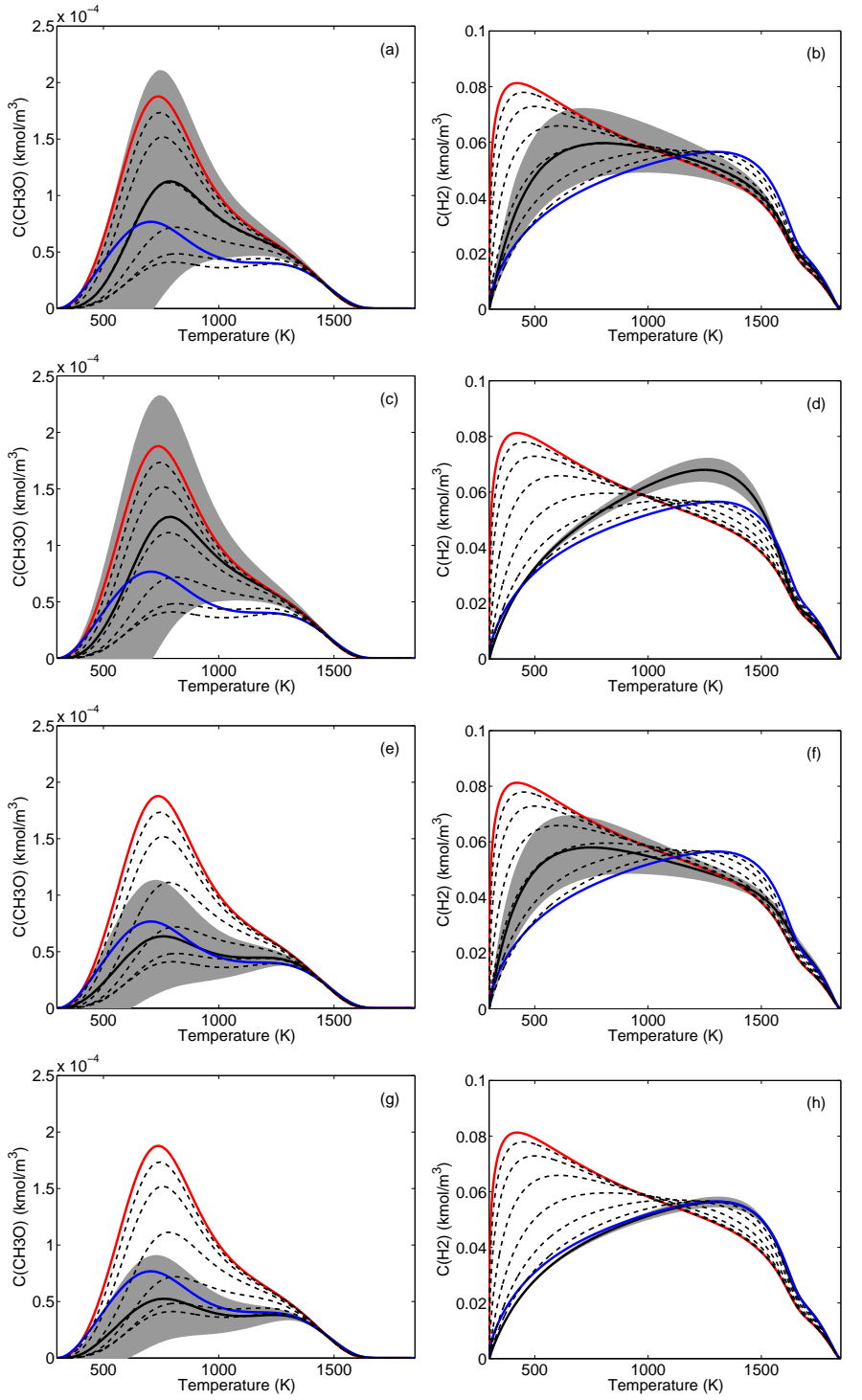

Figure 14: Conditional means of molar concentration (part 1) from the diffusion study cases for $\mathrm{CH}_{3} \mathrm{O}$ (left) and $\mathrm{H}_{2}$ (right); see figure 13 for details. 